\title{
Utilizando Métricas de Qualidade na Avaliação de uma Aplicação para Cidades Inteligentes
}

\author{
Alexandre R. Aragão ${ }^{1}$, Nathyane J. P. Moreno ${ }^{1}$, Davi Viana ${ }^{1}$, Francisco Silva ${ }^{1}$, \\ Tercio Sousa $^{1,2}$, Luis Rivero ${ }^{1}$, Ariel Teles ${ }^{1,2}$, Arlindo da Conceição ${ }^{3}$, Inaldo Costa ${ }^{4}$ \\ ${ }^{1}$ Universidade Federal do Maranhão (UFMA) - São Luís - MA - Brasil \\ ${ }^{2}$ Instituto Federal do Maranhão (IFMA) - Maranhão - Brasil \\ ${ }^{3}$ Universidade Federal de São Paulo (UNIFESP) - São José dos Campos - SP - Brasil \\ ${ }^{4}$ Instituto Tecnológico de Aeronáutica (ITA) - São José dos Campos - SP - Brasil \\ \{alexandre.aragao, nathyane.moreno, davi.viana,fssilva\}@lsdi.ufma.br \\ ariel.teles@ifma.edu.br, arlindo.conceicao@unifesp.br, inaldo@ita.br
}

\begin{abstract}
Several software applications have been modifying the human's way of life in the technological and social aspects. A certain set of applications supports the creation of Smart Cities (SM). Like any other type of application, they need an assessment in order to guarantee an adequate software quality level and to provide the best experience to users. However, applications in the smart cities context can include more technological characteristics than a traditional application, such as interoperability and context-changing according to the modification of the user environment. This paper presents a study with regard to two software quality characteristics for SM (context-awareness and calmness). Moreover, we present a discussion of such characteristics and their metrics in relation to human aspects of software application interaction.
\end{abstract}

Resumo. Diversas aplicações de software vêm modificando o estilo de vida das pessoas em aspectos tecnológicos e sociais. Um conjunto dessas aplicações possibilitam a criação das chamadas Cidades Inteligentes (CIs). Assim como qualquer outro tipo de aplicação, estas aplicações precisam ser avaliadas para garantir uma qualidade adequada e fornecer uma melhor experiência aos usuários. Contudo, aplicações no contexto de CIs podem conter características tecnológicas específicas que não são avaliadas em aplicações tradicionais, tais como aspectos de interoperabilidade e mudança de contexto da aplicação de acordo com a alteração do ambiente do usuário. Desta forma, este artigo apresenta um estudo sobre a avaliação de duas características de qualidade relevantes para CIs (sensibilidade ao contexto e calmness). Adicionalmente, apresentamos uma discussão sobre essas características e suas métricas em relação aos aspectos humanos da interação com a aplicação.

\section{Introdução}

O uso de smartphones e aplicativos que facilitam a vida cotidiana do ser humano cresce constantemente [AgBrasil 2019]. Essas aplicações buscam auxiliar diversas atividades e otimizar o tempo das pessoas. Concomitantemente, verifica-se a expansão dos centros urbanos que precisam lidar com desafios de planejamento, desenvolvimento e operações 
das cidades. O uso de aplicações e dispositivos móveis possui um papel fundamental nesta expansão urbana, pois estes auxiliam a gestão das cidades com soluções adequadas e otimizadas [Hernández-Muñoz et al. 2011], como serviços baseados em localização.

O uso de dispositivos móveis e infraestrutura tecnológica na gestão das cidades possibilita a criação das chamadas Cidades Inteligentes (CIs) [Chourabi et al. 2012]. De acordo com [Bakıcı et al. 2013], uma CI é uma "cidade intensiva e avançada de alta tecnologia que conecta pessoas, informações e elementos da cidade usando novas tecnologias para criar uma cidade sustentável e mais verde, comércio competitivo e inovador e um aumento na qualidade de vida" . Do ponto de vista computacional, CIs são um conglomerado de tecnologias para auxiliar na gestão das cidades, como: Internet das Coisas (IoT), computação ubíqua, big data, computação distribuída, redes de sensores e atuadores [Atzori et al. 2010, Jagadish et al. 2014]

Como qualquer outra aplicação de software, aplicações desenvolvidas para o contexto de IoT e CIs precisam ser avaliadas para executar suas funcionalidades adequadamente. Todavia, as abordagens tradicionais de avaliação de aplicações de software não consideram especificações de aplicações para estes contextos [Zambonelli 2017, Larrucea et al. 2017]. Essas aplicações devem ser adequadas para que qualquer pessoa destes centros urbanos se sinta à vontade para utilizá-la. Segundo [Carvalho et al. 2018], duas características importantes para aplicações no contexto de Sistemas Ubíquos são Sensibilidade ao Contexto (do inglês, Context Awareness) e Calmness.

A sensibilidade ao contexto é definida como a capacidade de perceber o sistema de informações contextuais e adaptar proativamente suas funcionalidades. Ou seja, ela visa tratar da possibilidade das aplicações de identificar as informações do contexto do sistema e dos usuários, para inferirem a situação em que esses encontram-se e, assim, proporcionarem adaptações eficientemente [Dey 2001]. Tal definição é relevante para CIs, pois analisam o grau de adaptação das aplicações ao contexto dos usuários, influenciando a tomada de decisão nas suas rotinas diárias. A característica de Calmness se refere à capacidade do sistema de auxiliar as atividades do usuário na hora e no local correto, fornecendo o melhor serviço possível [Riekki et al. 2004], acarretando portanto, um grande impacto na satisfação do usuário e, por fim, em sua avaliação.

Neste trabalho é apresentada uma análise dessas características e como elas podem ser empregadas para avaliação da qualidade de aplicações no contexto de CIs. Para isto, buscou-se na literatura definições dessas características e realizou-se um estudo de caso em uma aplicação real. Como resultado, identificou-se que as características avaliadas são adequadas para a avaliação da interação humano-computador para aplicações de CIs. Adicionalmente, observou-se a necessidade de avaliação de outras características, como Funcionalidade, Confiabilidade e Eficiência [Koscianski and Soares 2007].

Além desta seção introdutória, o restante do artigo está organizado em mais quatro seções. A Seção 2 apresenta o referencial teórico e os trabalhos relacionados sobre avaliação de qualidade para aplicações para IoT e CIs. A Seção 3 apresenta a descrição das métricas definidas na literatura e utilizadas neste trabalho. A Seção 4 apresenta a condução da pesquisa, os resultados e discussão dos resultados obtidos. A Seção 5 apresenta as considerações finais e trabalhos futuros. 


\section{Avaliação de Qualidade em IoT e CIs}

Uma CI é uma cidade inovadora que utiliza as Tecnologias de Informação e Comunicação (TIC), IoT e outros meios para melhorar a qualidade de vida, eficiência das operações e serviços urbanos ${ }^{1}$.

A partir dessa definição, verifica-se que uma CI deve: (a) proporcionar uma alta qualidade de vida aos cidadãos, integrando-os à cidade; (b) monitorar e/ou controlar condições, estados e informações dos diversos sensores e infraestruturas que compõem uma cidade. Com base nessas informações, uma aplicação para CI deve auxiliar seus usuários (e.g., cidadãos em suas atividades diárias) e/ou auxiliar na gestão da cidade.

Neste contexto, o paradigma de IoT é fundamental, visto que o uso de tecnologias e soluções desenvolvidas a partir dele é necessário para viabilizar uma CI [Chourabi et al. 2012, Hernández-Muñoz et al. 2011]. As avaliações de aplicações para CIs podem ser semelhantes àquelas para IoT, uma vez que compartilham características e necessidades comuns. Portanto, algumas métricas a serem usadas para avaliação de aplicações de IoT podem ser utilizadas para avaliar uma aplicação para CIs. Métricas como tempo de conexão com a Internet, tempo de alteração de rede, e grau de operação de rede não devem ser utilizadas na avaliação, visto que estão mais relacionadas ao dispositivo em que a aplicação está executando. Por outro lado, o tempo que esta aplicação leva para obter algum dado do banco de dados deve ser medido e avaliado.

Pesquisas referentes à avaliação de qualidade de aspectos específicos de sistemas complexos, como aplicações de IoT, CIs e Ubíquas têm sido desenvolvidas [Arasteh et al. 2016]. Todavia, por ser uma vertente tecnológica relativamente recente, ainda não há um conjunto sólido de abordagens em Engenharia de Software e Qualidade de Software [Zambonelli 2017, Larrucea et al. 2017]. Alguns trabalhos buscam analisar aplicações de IoT e sistemas ubíquos que podem ser utilizadas no contexto de CIs. O trabalho realizado em [Darin et al. 2016] apresenta a aplicação de diversas características, entre elas Sensibilidade ao Contexto e Calmness, para a avaliação de uma aplicação móvel de IoT. A aplicação é baseada baseada em proximidade para automatizar a criação de grupos virtuais e facilitar o compartilhamento de arquivos e criação de lista de presença durante reuniões [Darin et al. 2016], facilitando reuniões e interações entre os participantes. Os autores executaram um estudo para validação das métricas reunidas. Este estudo foi conduzido em um ambiente controlado.

A pesquisa realizada em [Carvalho et al. 2018] reuniu métricas para soluções de software relacionadas a sistemas ubíquos encontrados na literatura, partindo-se do trabalho anterior [Maia et al. 2016]. Nesta pesquisa, são reunidas as características e suas respectivas métricas, assim como foi selecionado e melhorado um conjunto de métricas específicas de aplicações móveis ubíquas, em que foram avaliadas três aplicações.

\section{Métricas para Avaliação de Sensibilidade ao Contexto e Calmness}

As características e métricas selecionadas para o estudo de caso foram obtidas a partir de [Carvalho et al. 2018]. Essas características estão relacionadas com aspectos humanos de interação com as aplicações, uma vez que mudanças repentinas nas aplicações podem influenciar no comportamento de seus usuários. A Tabela 1 apresenta as métricas para sensibilidade ao contexto.

\footnotetext{
${ }^{1}$ https://www.itu.int/en/ITU-T/focusgroups/ssc/Pages/default.aspx
} 
Tabela 1. Métricas selecionadas para avaliação de sensibilidade ao contexto.

\begin{tabular}{|c|c|c|c|c|}
\hline Nome & Propósito & Método & Fórmula & Interpretação \\
\hline $\begin{array}{l}\text { Exatidão de } \\
\text { informação } \\
\text { de contexto }\end{array}$ & $\begin{array}{l}\text { Quantidade de } \\
\text { informações de } \\
\text { contexto que foram } \\
\text { coletadas } \\
\text { corretamente }\end{array}$ & $\begin{array}{l}\text { Log de } \\
\text { interação do } \\
\text { usuário e } \\
\text { observação }\end{array}$ & $\begin{array}{l}\mathrm{X}=\frac{\sum_{i=1}^{N}\left(\frac{\mathbf{A}}{\mathbf{B}}\right)}{N} \cdot 100 \\
\mathbf{N}=\text { Número de tipos de } \\
\text { informações de contexto } \\
\mathbf{A}=\text { Número de } \\
\text { informações de contexto } \\
\text { corretamente medidas. } \\
\mathbf{B}=\text { Número de } \\
\text { informações e contexto } \\
\text { medidas }\end{array}$ & $\begin{array}{l}\text { Quanto mais } \\
\text { perto de } \\
100 \% \text {, melhor }\end{array}$ \\
\hline Granularidade & $\begin{array}{l}\text { Nível de } \\
\text { detalhamento das } \\
\text { informações de } \\
\text { contexto. }\end{array}$ & Questionário & $\mathbf{X}=\mathrm{A}$, onde $\mathrm{A} \in\{1,2,3\}$ & $\begin{array}{l}\text { (1) Baixo: } \\
\text { Informação } \\
\text { de baixo } \\
\text { nível; } \\
\text { (2) Médio: } \\
\text { Situação de } \\
\text { contexto; } \\
\text { (3) Alto: } \\
\text { Informação } \\
\text { de alto nível. }\end{array}$ \\
\hline $\begin{array}{lr}\text { Grau } & \text { de } \\
\text { adaptação } & \text { à } \\
\text { mudança } & \text { de } \\
\text { contexto } & \end{array}$ & $\begin{array}{l}\text { Nível de adaptação } \\
\text { de funcionalidades } \\
\text { referentes às } \\
\text { mudanças de } \\
\text { contexto. }\end{array}$ & $\begin{array}{l}\log \text { de } \\
\text { interação do } \\
\text { usuário e } \\
\text { observação }\end{array}$ & $\begin{array}{l}\mathrm{X}=\frac{\sum_{i=1}^{N}\left(\frac{\mathrm{A}}{\mathrm{B}}\right)}{N} \cdot 100 \\
\mathbf{N}=\text { Número de tipos de } \\
\text { adaptações } \\
\mathbf{A}=\text { Número de vezes que } \\
\text { os sistema se adaptou } \\
\mathbf{B}=\text { Número de vezes que } \\
\text { uma adaptação foi acionada }\end{array}$ & $\begin{array}{l}\text { Quanto mais } \\
\text { perto de } \\
100 \% \text {, melhor }\end{array}$ \\
\hline $\begin{array}{l}\text { Exatidão de } \\
\text { adaptação }\end{array}$ & $\begin{array}{l}\text { Medida que intenta } \\
\text { verificar se as } \\
\text { adaptações foram } \\
\text { realizadas de forma } \\
\text { eficiente. }\end{array}$ & $\begin{array}{l}\log \text { de } \\
\text { interação do } \\
\text { usuário e } \\
\text { observação }\end{array}$ & $\begin{array}{l}\mathrm{X}=\frac{\sum_{i=1}^{N}\left(\frac{\mathrm{A}}{\mathrm{B}}\right)}{N} \cdot 100 \\
\mathbf{N}=\text { Número de tipos de } \\
\text { adaptações. } \\
\mathbf{A}=\text { Número de adaptações } \\
\text { corretamente realizadas. } \\
\mathbf{B}=\text { Número de adaptações } \\
\text { realizadas. }\end{array}$ & $\begin{array}{l}\text { Quanto mais } \\
\text { perto de } \\
100 \% \text {, melhor }\end{array}$ \\
\hline $\begin{array}{l}\text { Frequência de } \\
\text { mudança de } \\
\text { contexto }\end{array}$ & $\begin{array}{l}\text { Intenta verificar a } \\
\text { frequência com que } \\
\text { as mudanças de } \\
\text { contexto ocorrem. }\end{array}$ & $\begin{array}{l}\log \text { de } \\
\text { interação do } \\
\text { usuário }\end{array}$ & $\begin{array}{l}\mathbf{X}=\frac{\sum_{i=1}^{N}\left(\frac{\mathbf{A}}{\mathbf{B}}\right)}{N} \\
\mathbf{A i}=\text { Tempo de uma } \\
\text { mudança a outra } \\
\mathbf{N}=\mathrm{N} \text { intervalos de medida }\end{array}$ & $\begin{array}{l}\text { Baixa: minu- } \\
\text { tos; } \\
\text { Média:segundos } \\
\text { Alta: milise- } \\
\text { gundos. }\end{array}$ \\
\hline $\begin{array}{ll}\text { Tempo de } \\
\text { adaptação }\end{array}$ & $\begin{array}{l}\text { Tempo necessário } \\
\text { para que uma } \\
\text { adaptação ocorra } \\
\text { desde uma mudança } \\
\text { de contexto. }\end{array}$ & $\begin{array}{l}\log \text { de } \\
\text { interação do } \\
\text { usuário }\end{array}$ & $\begin{array}{l}\mathbf{X}=\mathrm{T} \text {, onde } \mathrm{T} \text { é a média do } \\
\text { tempo que o sistema leva } \\
\text { para se adaptar desde uma } \\
\text { mudança de contexto. }\end{array}$ & $\begin{array}{l}\text { Baixo: mili- } \\
\text { segundos; } \\
\text { Médio: se- } \\
\text { gundos; } \\
\text { Alto: min. }\end{array}$ \\
\hline
\end{tabular}

Calmness é uma característica centrada no usuário, suas métricas são definidas a partir da perspectiva do usuário. A avaliação deve ser feita pelos usuários reais em uma situação de uso real. Eles devem testar o aplicativo e avaliar a Calmness com base em sua experiência [Riekki et al. 2004]. 
A Tabela 2 apresenta as métricas para Calmness. Adicionalmente, Calmness utiliza duas métricas definidas na característica de sensibilidade ao contexto, são elas "Exatidão da informação de contexto" e "Grau de adaptação à mudança de contexto".

Tabela 2. Métricas selecionadas para avaliação de Calmness.

\begin{tabular}{|c|c|c|c|c|}
\hline Nome & Propósito & Método & Fórmula & Interpretação \\
\hline $\begin{array}{l}\text { Aplicação } \\
\text { proativa }\end{array}$ & $\begin{array}{l}\text { Requisitos que } \\
\text { podem ser } \\
\text { automatizados pelo } \\
\text { sistema em relação } \\
\text { às ações do usuário. }\end{array}$ & $\begin{array}{l}\text { Questionário } \\
\text { e Observação }\end{array}$ & $\begin{array}{l}\mathbf{X}=\left(\frac{\mathbf{A}_{\mathbf{i}}}{\mathbf{A}_{\mathbf{i}}+\mathbf{N}_{\mathbf{i}}}\right) \cdot 100 \\
\mathbf{A i}=\text { Número de } \\
\text { informações de contexto } \\
\text { automatizadas pelo sistema } \\
\mathbf{N}=\text { Número de requisitos }\end{array}$ & $\begin{array}{l}\text { Quanto mais } \\
\text { perto de } \\
100 \% \text {, melhor }\end{array}$ \\
\hline $\begin{array}{l}\text { Grau de dis- } \\
\text { ponibilidade }\end{array}$ & $\begin{array}{l}\text { Se a aplicação estava } \\
\text { disponível em } \\
\text { qualquer lugar e a } \\
\text { qualquer momento. }\end{array}$ & Questionário & $\mathbf{X}=\mathrm{A}$, onde $\mathrm{A} \in\{1,2,3,4\}$ & $\begin{array}{l}\text { (1) Alta; } \\
\text { (2) Média; } \\
\text { (3) Baixa; } \\
\text { (4) Muito } \\
\text { baixa. }\end{array}$ \\
\hline $\begin{array}{l}\text { Grau de } \\
\text { sincronização } \\
\text { de sensibi- } \\
\text { lidade de } \\
\text { contexto }\end{array}$ & $\begin{array}{l}\text { Impressão do usuário } \\
\text { em relação às } \\
\text { interações com a } \\
\text { aplicação. }\end{array}$ & Questionário & $\mathbf{X}=\mathrm{A}$, onde $\mathrm{A} \in\{1,2,3,4\}$ & $\begin{array}{l}\text { (1) Alto; } \\
\text { (2) Médio; } \\
\text { (3) Baixo; } \\
\text { (4) Muito } \\
\text { baixo. }\end{array}$ \\
\hline $\begin{array}{ll}\text { Grau } & \text { de } \\
\text { cortesia } & \text { de } \\
\text { interação } & \end{array}$ & $\begin{array}{l}\text { A aplicação } \\
\text { requisitou a atenção } \\
\text { do usuário somente } \\
\text { quando necessário. }\end{array}$ & Questionário & $\mathbf{X}=\mathrm{C}$, onde $\mathrm{C} \in\{1,2,3,4\}$ & $\begin{array}{l}\text { (1) Alto; } \\
\text { (2) Médio; } \\
\text { (3) Baixo; } \\
\text { (4) Muito } \\
\text { baixo. }\end{array}$ \\
\hline $\begin{array}{ll}\text { Grau de } & \text { re- } \\
\text { levância } & \text { de } \\
\text { interação } & \end{array}$ & $\begin{array}{l}\text { A aplicação } \\
\text { requisitou e mostrou } \\
\text { informações úteis da } \\
\text { percepção do } \\
\text { usuário. }\end{array}$ & Questionário & $\mathbf{X}=\mathrm{D}$, onde $\mathrm{D} \in\{1,2,3,4\}$ & $\begin{array}{l}\text { (1) Alto; } \\
\text { (2) Médio; } \\
\text { (3) Baixo; } \\
\text { (4) Muito } \\
\text { baixo. }\end{array}$ \\
\hline $\begin{array}{l}\text { Quantidade } \\
\text { de mudança } \\
\text { de foco }\end{array}$ & $\begin{array}{l}\text { Quantidade de ações } \\
\text { que um usuário toma } \\
\text { para que a aplicação } \\
\text { funcione } \\
\text { corretamente e que } \\
\text { mudam o foco de sua } \\
\text { atividade principal. }\end{array}$ & Observação & $\begin{array}{l}\mathbf{X}=\mathrm{A}, \text { onde } \\
\mathrm{A}=\text { Número de ações que } \\
\text { mudam o foco do usuário } \\
\text { durante o uso da aplicação }\end{array}$ & $\begin{array}{l}\text { Quanto mais } \\
\text { longe de zero, } \\
\text { melhor }\end{array}$ \\
\hline $\begin{array}{l}\text { Tempo médio } \\
\text { entre falhas }\end{array}$ & $\begin{array}{l}\text { Tempo entre falhas } \\
\text { ocorridas durante o } \\
\text { uso da aplicação. }\end{array}$ & $\begin{array}{l}\log \text { de } \\
\text { interação do } \\
\text { usuário e } \\
\text { observação }\end{array}$ & $\begin{array}{l}\mathrm{X}=\left(\frac{\mathbf{Y}}{Z}\right) \cdot \\
\mathbf{Y}=\text { Tempo de operação } \\
\mathbf{Z}=\text { Número de falhas } \\
\text { detectadas }\end{array}$ & $\begin{array}{l}\text { Quanto maior, } \\
\text { melhor }\end{array}$ \\
\hline
\end{tabular}

\section{Utilização das Métricas na Avaliação de Aplicação de CIs}

O objetivo deste estudo foi analisar as duas características e suas métricas na avaliação de qualidade de uma aplicação para CIs. A seguir, apresenta-se a aplicação e o planejamento da avaliação. 


\subsection{Aplicação para CIs e planejamento da avaliação}

Para realizar a avaliação das métricas e da metodologia proposta, foi utilizada uma aplicação desenvolvida no Laboratório de Sistemas Distribuídos Inteligentes da Universidade Federal do Maranhão. Essa aplicação é voltada para o monitoramento de frotas de veículos utilizando smartphones. Dentre as principais funcionalidades da aplicação estão: monitoramento da velocidade de cada veículo da frota; detecção da localização dos veículos exibida em um mapa em tempo real; acompanhamento de um histórico de paradas do veículo (quando o veículo atingir a velocidade zero); e alertas sobre solicitações de pedidos de ajuda feitos pelo motorista.

A aplicação divide-se em dois módulos: o primeiro, denominado Mobile Vehicle Monitoring System (MVMS), que executa em dispositivos móveis com a plataforma Android, os quais ficam embarcados nos veículos da frota. Este módulo captura os dados de contexto a partir dos sensores do smartphone (acelerômetro e o sensor que detecta o nível de bateria) e GPS. A partir do GPS é obtida a coordenada geográfica (latitude e longitude) do veículo que serão utilizadas para o monitoramento da sua localização.

Para detectar a velocidade com que o veículo está se deslocando e identificar que realizou-se uma parada, foi utilizada uma API do Google Android ${ }^{2}$. O nível de bateria do smartphone é um outro dado de contexto capturado e utilizado para ajustar a frequência que as demais informações são enviadas através de uma conexão com a Internet para o outro componente da arquitetura. O objetivo deste controle é economizar a bateria do dispositivo móvel à medida em que seu nível diminui. A Figura 1(a) mostra a interface gráfica do módulo MVMS e exibe os dados de contexto capturados do dispositivo móvel.

O outro módulo da aplicação, denominado Vehicle Tracking Center (VTC), tem por objetivo manter uma central de monitoramento de todos veículos da frota. A Figura 1(b) e (c) mostram as interfaces gráfica do módulo VTC. O módulo permanece obtendo os dados de contexto enviados pelo MVMS, e assim que os recebe, atualiza a interface gráfica de acordo com as informações de contexto recebidas. Por exemplo, na Figura 1(c) é exibido um histórico de paradas, que é atualizado cada vez que for detectado que a velocidade de algum dos veículos foi igual a zero.

\subsection{Planejamento do Estudo}

Inicialmente, elaborou-se um cenário de utilização da aplicação. Neste cenário, definiuse que dois usuários executariam a aplicação, logo, o estudo foi realizado em pares. Um usuário monitorava com o VTC (versão de aplicativo móvel) e o outro usuário percorreria uma rota previamente escolhida em algum veículo disponível. Posteriormente, foi feita a inversão dos papéis dos usuários, para que eles pudessem utilizar os dois módulos e avaliar a aplicação completa.

Por fim, um questionário ${ }^{3}$ foi entregue após o estudo para ser respondido pelos usuários e também pelo desenvolvedor da aplicação. Esse questionário foi necessário para obter alguns dados relacionados às métricas. Algumas métricas foram coletadas diretamente a partir de dados da aplicação e dispositivo móvel. A aplicação foi ajustada para prover dados necessários que foram utilizadas para os cálculos das métricas. $\mathrm{O}$ desenvolvedor da aplicação também apoiou a execução do estudo e coleta das métricas.

\footnotetext{
${ }^{2}$ Location do Google Play Services.

${ }^{3}$ http://goo.gl/nfAj77
} 


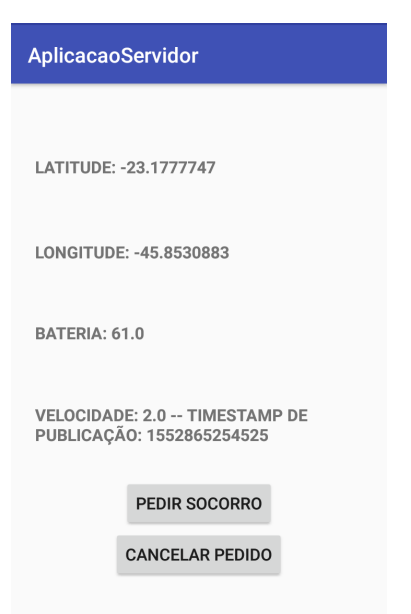

(a) Módulo MVMS.

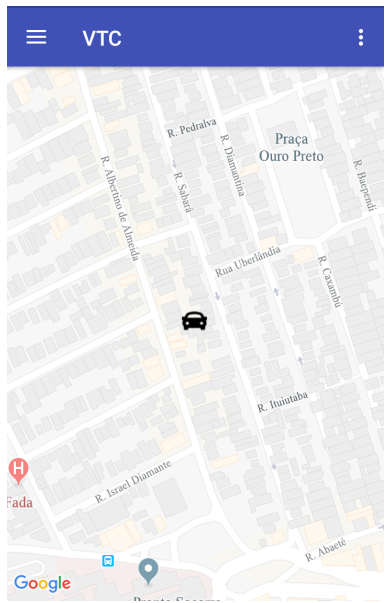

(b) Módulo VTC exibindo veículo no mapa.

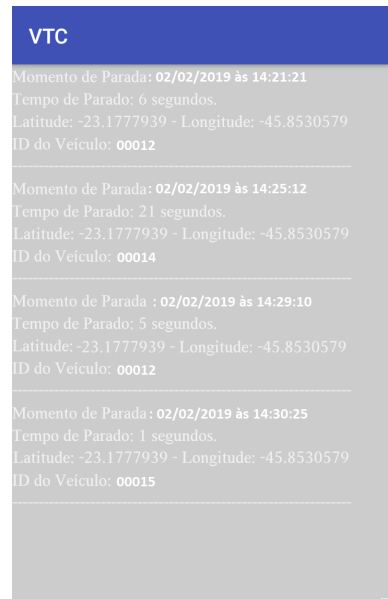

(c) Módulo VTC exibindo o monitoramento de paradas.

Figura 1. Capturas de tela dos módulos MVMS e VTC.

\subsection{Execução e Resultados}

Oito participantes foram selecionados para uso da aplicação e execução do cenário. Cada par de usuários demorou entre três e seis minutos para executar o cenário proposto. $\mathrm{O}$ desenvolvedor da aplicação também forneceu dados para uma métrica (granularidade de contexto). Em seguida, os dados foram tabulados pelos pesquisadores. Os resultados são apresentados nas Tabelas 3 e 4.

Em relação à característica de sensibilidade ao contexto, verificou-se que a métrica exatidão de informação de contexto obteve um resultado satisfatório. Cabe ressaltar que a conexão intermitente e a fraca conectividade do dispositivo com a rede de telefonia móvel para acessar a Internet pode interferir na recepção e/ou envio dos dados de contexto, influenciando a taxa de atualização ou atualidade da posição no mapa.

Tabela 3. Resultados das métricas para sensibilidade ao contexto.

\begin{tabular}{lll}
\hline Medida & Dado Coletado & Resultado \\
\hline $\begin{array}{l}\text { Exatidão de informação de } \\
\text { contexto }\end{array}$ & $\begin{array}{l}\text { Posição no mapa } \\
\text { Velocidade } \\
\text { Nível da bateria }\end{array}$ & $90 \%$ \\
\hline Granularidade de contexto & Questão 1 do questionário de desenvolvedor & Alto \\
\hline $\begin{array}{l}\text { Grau de adaptação à } \\
\text { mudança de contexto }\end{array}$ & $\begin{array}{l}\text { Localização nos pontos marcados para solicitação } \\
\text { de socorro após mudar de local } \\
\text { Mostrar o tempo de parada após movimentar-se } \\
\text { novamente } \\
\text { Veículo muda de cor } \\
\text { Nível da bateria alto/médio/baixo }\end{array}$ & \\
& $\begin{array}{l}\text { Localização de acordo com posição real - usuário } \\
\text { Dados coletados de acordo com nível de bateria }\end{array}$ & \\
\hline Exatidão de adaptação & & \\
\hline
\end{tabular}


A granularidade de contexto foi avaliada como alta pelo desenvolvedor da aplicação, sendo um resultado adequado já que os dados necessários para se obter a localização, nível de bateria e velocidade dependem do smartphone, culminando em uma aplicação com menos mudanças bruscas devido ao contexto. Deve-se ressaltar que a granularidade de contexto pode interferir no desempenho da aplicação e no comportamento do usuário.

O grau de adaptação à mudança de contexto mostra-se aceitável, já que normalmente a aplicação exibe o dispositivo (que possui o MVMS) em movimento de acordo com a velocidade mostrada na tela do VTC. Quanto a exatidão de adaptação, o valor referente atende à demanda dos dados coletados, porém a adaptação referente à bateria não se mostrou perceptível devido não a chegar a haver mudança na frequência durante a avaliação ( $3 \mathrm{~s}, 5 \mathrm{~s}$ e $8 \mathrm{~s}$ para os níveis de baterias alto, médio e baixo, respectivamente). Todavia verifica-se um valor médio para esta métrica (55\%). Não foi possível coletar duas métricas sobre esta característica, são elas Frequência de mudança de contexto e Tempo de adaptação, uma vez que a conexão à Internet para comunicação do MVMS e VTC interferia no resultado. Essas não foram utilizadas neste trabalho, pois a coleta de dados foi inviabilizada pela aplicação móvel.

Em relação à característica Calmness, observou-se que as métricas de grau de disponibilidade, grau de sincronização de sensibilidade ao contexto, grau de cortesia de interação, grau de relevância de interação obtiveram um resultado satisfatório. A aplicação forneceu o serviços de localização, pedidos de socorro e verificação de paradas. Esses serviços foram prestados durante a comunicação entre os módulos MVMS e o VTC da aplicação móvel, sem perturbações desnecessárias aos usuários, uma vez que houve conexão com a Internet.

Tabela 4. Resultados das métricas para Calmness

\begin{tabular}{|c|c|c|}
\hline Medida & Dado Coletado & Resultado \\
\hline Grau de disponibilidade & Disponibilidade da aplicação para o usuário & Alto \\
\hline $\begin{array}{l}\text { Grau de sincronização de } \\
\text { sensibilidade ao contexto }\end{array}$ & Interação no momento correto para o usuário & Alto \\
\hline Grau cortesia de interação & Nível de atenção de interação para o usuário & Médio \\
\hline $\begin{array}{l}\text { Grau de relevância de } \\
\text { interação }\end{array}$ & $\begin{array}{l}\text { Nível de importância de informações apresentadas } \\
\text { ou requisitadas para o usuário }\end{array}$ & Médio \\
\hline Tempo médio entre falhas & Falhas durante uso da aplicação & 2 minutos \\
\hline $\begin{array}{l}\text { Quantidade de mudança de } \\
\text { foco }\end{array}$ & $\begin{array}{l}\text { Ações de configurações de GPS e Internet, reini- } \\
\text { ciar a aplicação. }\end{array}$ & $\begin{array}{l}2 \text { ações do } \\
\text { usuário }\end{array}$ \\
\hline $\begin{array}{l}\text { Grau de adaptação à } \\
\text { mudança de contexto }\end{array}$ & $\begin{array}{l}\text { Localização nos pontos marcados para solicitação } \\
\text { de socorro após mudar de local } \\
\text { Tempo de parada após movimentar-se novamente } \\
\text { Veículo muda de cor } \\
\text { Nível da bateria alto } \backslash \text { médio } \backslash \text { baixo }\end{array}$ & $88 \%$ \\
\hline Exatidão de adaptação & $\begin{array}{l}\text { Localização apresentada de acordo com a posição } \\
\text { real do usuário } \\
\text { Dados coletados de acordo com nível de bateria }\end{array}$ & $55 \%$ \\
\hline
\end{tabular}


Quanto ao tempo médio entre falhas, observou-se que a aplicação parava de funcionar devido à rotação da tela ou problemas de programação da aplicação identificadas a partir do uso da aplicação e do dispositivo móvel. Por fim, em relação à métrica quantidade de mudança de foco, verificou-se que a ação de ativar o GPS e a conexão Internet $(3 \mathrm{~g} / 4 \mathrm{~g})$ para que o serviço funcionasse, fazia com que o usuário precisasse reiniciar a aplicação para que suas funcionalidades fossem executadas adequadamente. $\mathrm{O}$ resultado para grau de adaptação à mudança de contexto e exatidão de informação de contexto são os mesmos coletados para sensibilidade ao contexto. A métrica de aplicação proativa não foi coletada nesta pesquisa, pois as informações sobre sua interpretação não possuíam detalhes suficientes para explica-la, principalmente sobre as ações do usuário que devem ser consideradas na avaliação.

\subsection{Discussão}

A utilização de métricas para avaliar as características de sensibilidade ao contexto e Calmness para avaliar aplicações no contexto de CIs mostrou-se relevante nesta pesquisa. Algumas métricas foram coletadas a partir de $\log s$ de funcionalidades da aplicação. Devido à aplicação ter sido desenvolvida no mesmo laboratório desta pesquisa, conseguiu-se solicitar ao desenvolvedor que nos fornecesse alguns dados para calcular as métricas. Em caso de aplicações prontas e desenvolvidas por terceiros, seria difícil conseguir analisar determinados eventos e interações com a aplicação.

Com os dados coletados, foi possível analisar como a aplicação poderia impactar positiva ou negativamente no comportamento do usuário. As características de sensibilidade ao contexto e Calmness fornecem indicativos de como a aplicação pode influenciar o comportamento dos usuários. Este tipo de avaliação de qualidade é relevante para que as aplicações sejam as mais adequadas possíveis do ponto de vista do usuário. Como limitações do trabalho, observa-se que coletar as métricas apenas no uso de uma aplicação não fornece indícios suficientes para analisar de maneira abrangente todas métricas estudadas neste trabalho. Além disso, há limitação da aplicação em si em relação aos dados necessários de coleta. Os desenvolvedores podem desconhecer determinadas métricas e isso dificulta a geração e coleta dos dados para análise.

\section{Considerações Finais e Trabalhos Futuros}

Neste trabalho foram apresentados os resultados obtidos da avaliação de qualidade de uma aplicação para CIs. Para isso, buscou-se na literatura características e métricas relevantes para aplicações deste contexto.

A aplicação avaliada obteve resultados satisfatórios para a maioria das medidas de ambas características. Essas características podem ser utilizadas para analisar aspectos da interação do usuário com as aplicações, uma vez que elas podem despertar determinadas reações nos usuários. Por exemplo, a rápida mudança de contexto pode levar a níveis de irritação do usuário. Os resultados obtidos mostram indícios da viabilidade dessas métricas para avaliação de aplicações no contexto de cidades inteligentes.

A impossibilidade de coletar determinados dados, abre uma possibilidade de trabalho futuro, isto é, adaptar a métrica para melhor se adequar a aplicações deste contexto. Adicionalmente, é necessário identificar outras medidas para avaliar outras características das aplicações de CIs. Por fim, é preciso analisar como essas métricas se comportam ao avaliar aplicações interoperáveis. 


\section{Agradecimentos}

O presente trabalho foi realizado com apoio da Coordenação de Aperfeiçoamento de Pessoal de Nível Superior - Brasil (CAPES) - Código de Financiamento 001. Por fim, este trabalho teve o apoio da FAPEMA e UFMA.

\section{Referências}

AgBrasil (2019). Brasil é $5^{\circ}$ país em ranking de uso diário de celulares no mundo. Acessado em: 25 de fevereiro de 2019. Disponível em: http://agenciabrasil.ebc.com.br/geral/noticia/2019-01/brasil-foi-5o-pais-em-rankingde-uso-diario-de-celulares-no-mundo.

Arasteh, H., Hosseinnezhad, V., Loia, V., Tommasetti, A., Troisi, O., Shafie-Khah, M., and Siano, P. (2016). Iot-based smart cities: a survey. In 2016 IEEE 16th International Conference on Environment and Electrical Engineering (EEEIC), pages 1-6. IEEE.

Atzori, L., Iera, A., and Morabito, G. (2010). The Internet of Things: A survey. Computer Networks, 54(15):2787-2805.

Bakıc1, T., Almirall, E., and Wareham, J. (2013). A smart city initiative: the case of barcelona. Journal of the Knowledge Economy, 4(2):135-148.

Carvalho, R. M., de Castro Andrade, R. M., and de Oliveira, K. M. (2018). Aquarium - a suite of software measures for hci quality evaluation of ubiquitous mobile applications. Journal of Systems and Software, 136:101 - 136.

Chourabi, H., Nam, T., Walker, S., Gil-Garcia, J. R., Mellouli, S., Nahon, K., Pardo, T. A., and Scholl, H. J. (2012). Understanding smart cities: An integrative framework. In 2012 45th Hawaii international conference on system sciences, pages 2289-2297.

Darin, T., Barbosa, J., Rodrigues, B., and Andrade, R. (2016). Greatroom: Uma aplicação android baseada em proximidade para a criação de salas virtuais inteligentes. In Workshop de Ferramentas e Aplicações (WFA). WebMedia 2019.

Dey, A. K. (2001). Understanding and using context. Personal Ubi. Computing, 5(1):4-7.

Hernández-Muñoz, J. M., Vercher, J. B., Muñoz, L., Galache, J. A., Presser, M., Gómez, L. A. H., and Pettersson, J. (2011). Smart cities at the forefront of the future internet. In The future internet assembly, pages 447-462. Springer.

Jagadish, H., Gehrke, J., Labrinidis, A., Papakonstantinou, Y., Patel, J. M., Ramakrishnan, R., and Shahabi, C. (2014). Big data and its technical challenges. Communications of the ACM, 57(7):86-94.

Koscianski, A. and Soares, M. S. (2007). Qualidade de Software. Novatec, São Paulo, 2 edition.

Larrucea, X., Combelles, A., Favaro, J., and Taneja, K. (2017). Software engineering for the internet of things. IEEE Software, 34(1):24-28.

Maia, R., Andrade, R., Oliveira, K., Santos, I. D. S., and Bezerra, C. I. M. (2016). Quality characteristics and measures for human-computer interaction evaluation in ubiquitous systems. Software Quality Journal.

Riekki, J., Isomursu, P., and Isomursu, M. (2004). Evaluating the calmness of ubiquitous applications. volume 3009, pages 105-119.

Zambonelli, F. (2017). Key abstractions for iot-oriented software engineering. IEEE Software, (1):38-45. 